\title{
Megasource \\ time-domain electromagnetic sounding at Poggi del Sasso - Cinigiano (GR)
}

\author{
George V. Keller $\left({ }^{1}\right)$, Paolo Cantini $\left({ }^{2}\right)$, Raffaello Carrara $\left({ }^{3}\right)$, \\ Osvaldo Faggioni $\left({ }^{2}\right)$ and Eros Pinna $\left({ }^{2}\right)$ \\ ( $\left.{ }^{1}\right)$ Colorado School of Mines, Golden, CO, U.S.A. \\ ${ }^{2}{ }^{2}$ Dipartimento di Scienze della Terra, Università di Pisa, Italy \\ $\left.{ }^{3}\right)$ Dipartimento di Fisica, Università di Pisa, Italy
}

\begin{abstract}
An experiment was carried out in the vicinity of the «I Terzi» area in Southeastern Tuscany (fig. 1) to evaluate the applicability of the Time Domain Electromagnetic (TDEM) sounding method under the geological and environmental conditions prevailing in that area. An electromagnetic source was established using a motor-generator set and heavy cable. Measurements were attempted at four sites. Numerous samples of electromagnetic noise were recorded at each of these sites. At one site, signals transmitted for a grounded dipole source at 1.6 $\mathrm{km}$ distance were also recorded with the noise. The single set of observations has been processed and inverted to yield a six-layer piecewise constant resistivity depth-profile to a depth of about $2 \mathrm{~km}$. The primary achievement of the experiment was demonstration of the practicability of TDEM methods under the conditions pre-
vailing in the site.
\end{abstract}

Key words electromagnetism - resistivity - transient signal

\section{Introduction}

Time Domain Electromagnetic (TDEM) sounding is a method for determining the electrical resistivity structure of the earth. The TDEM method makes use of grounded cable to generate a controlled electromagnetic field and vertical-axis induction coil to measure the transmitted field at a location, or several locations, where knowledge of the elctrical character of the subsurface is desired (fig. 2) (Harthill, 1976; Keller et al., 1984).

The method is used to determine electrical properties at depths of only few tens of meters or of tens of kilometers. The depth to which the earth can be probed depends on the strength of the electromagnetic field generated by the source and on its frequency content. To probe to depths of many kilometers, it is more energized with a current square wave having an amplitude measured in hundreds or thousands of amperes, and a period measured in seconds or tens of seconds.

The signal recorded in carrying out a TDEM sounding is the time derivative of the magnetic field at a receiver site. This signal is a decaying transient with a shape determined by the electrical structure of the Earth. In routine surveys designed to achieve penetrations of a few kilometers or more, this transient is measured over a range in decay times from milliseconds to tens of seconds.

The TDEM method might well be applied effectively in the study of various important geological problems in Italy, including definition of geothermal systems and the evaluation of the hydrocarbon prospects for rocks beneath overthrust sheets. 


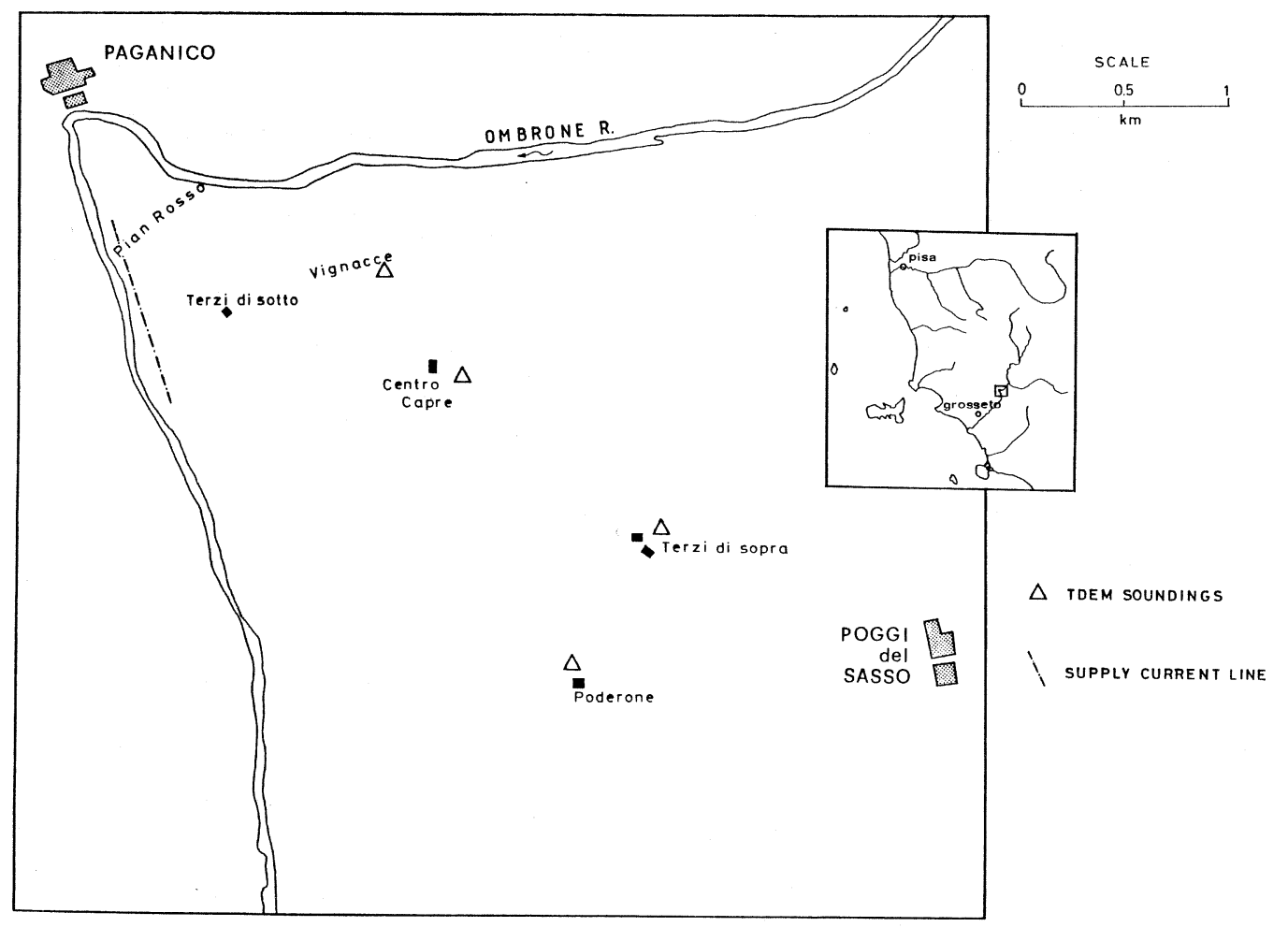

Fig. 1. Area of the experiment and location of TDEM soundings.
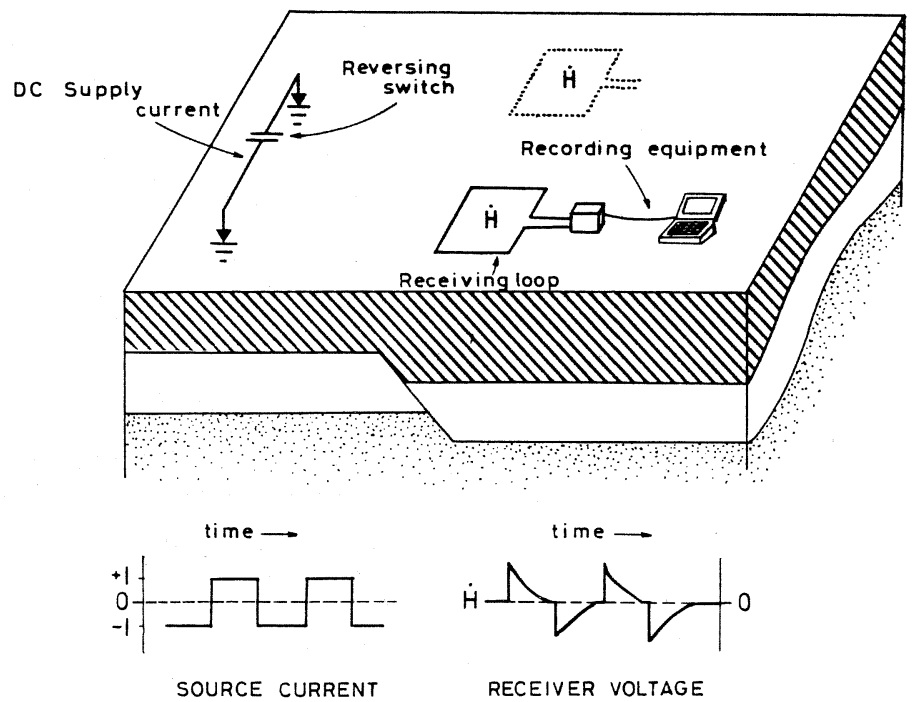

Fig. 2. Experimental equipment. 


\section{TDEM system theoretical elements}

On the assumption that the Earth is uniform, the vertical magnetic field at the sensor will be

$$
H_{2}=\frac{p}{4 \pi R^{2}} h_{2} \cos \beta
$$

where $p$ is the moment of the source, $R$ is the distance from the source to the receiver and the equatorial, $\beta$ is misalignment angle between the radius vector from the source to the receiver and the equatorial axis of the source, and

$$
h_{2}=\frac{3}{\sqrt{\pi}} \cdot \frac{e^{-u^{2}}}{u}+\left(1-\frac{3}{2 u^{2}}\right) \cdot \Phi(u)
$$

where

$$
u=\frac{2 \sqrt{\pi}}{\tau} \cdot R, \quad \tau=2 \pi \sqrt{\frac{2 t \rho}{\mu_{0}}}
$$

$\Phi(u)$ is the error function, and $t$ is the time following the beginning of the transient, $\rho$ is the resistivity of the medium and $\mu_{0}$ is the magnetic permeability.

The asymptotic behaviour of $H_{z}$ is

$$
H_{z}=-\frac{p}{4 \pi R^{2}} \cdot\left[\frac{6 t \rho}{R^{2} \mu_{0}}-1\right] \cdot \cos \beta
$$

for late times

$$
H_{z}=\frac{p \cdot \mu_{0}^{3 / 2} \cdot R}{60 \pi^{3 / 2} t^{3 / 2} \delta^{3 / 2}} \cdot \cos \beta
$$

If $B \approx \mu_{0} H$ :

$$
\frac{\partial B_{z}}{\partial t}=-\frac{3 p \rho}{2 \pi\left(R^{2}\right)^{2}} \cdot \cos \beta
$$

for early times,

$$
\frac{\partial B_{z}}{\partial t}=\frac{p \mu_{0}^{5 / 2} R}{40 \pi^{3 / 2} t^{5 / 2} \delta^{3 / 2}} \cdot \cos \beta
$$

for late times.
In conclusion we obtain a simple expression for the resistivity

$$
\begin{gathered}
\rho_{\text {early }}=K\left(R^{2}\right)^{2} V_{\text {meas }}(t) \\
\rho_{\text {late }}=K^{\prime} R^{2 / 3} t^{-5 / 3} V_{\text {meas }}(t)
\end{gathered}
$$

where $V(t)$ is the measured voltage (TDEM signal). In the case of two layers of conductivity $\sigma_{1}$ and $\sigma_{2}$ respectively, the contribution of the upper layer to the «apparent» conductivity is

$$
\sigma_{\mathrm{au}}=\sigma_{1}-\sigma_{1} R(z)=\sigma_{1}[1-R(z)]
$$

where $R(z)$ is the relative contribution to the apparent conductivity by all material below a depth $z$ (Harthill, 1976; Kaufman and Keller, 1983; Keller et al., 1984; Petry et al., 1989; Hördt et al., 1990).

\section{Equipment and experimental operations}

The source cable and electrodes were placed along the Ombrone river. Electrodes consisted of metal rods $2.5 \mathrm{~cm}$ in diameter and $60 \mathrm{~cm}$ in length. These were driven into soil for their full length. Contact with the soil was enhanced by dropping a few hundred grams of salt into each electrode hole. Six electrodes were placed at each end of the source cable.

The $50 \mathrm{~Hz}, 220 \mathrm{~V}$ output of the motor-generator set was used to operate a high capacity pulse former. This equipment rectified the output of the motor-generator, and switched the DC power at periodic intervals, set at $15 \mathrm{~s}$ for the demonstration. Switching was controlled by a simple relay for the demonstration, but in more extensive operations, switching would have been controlled with a high precision clock.

With this setup, a total current flow of $7.5 \mathrm{~A}$ through the cable was achieved, providing a current step amplitude of $15 \mathrm{~A}$ on switching the direct current from one polarity to the other. In full-scale operation, the level of the current steps could easily be increased to $200 \mathrm{~A}$ using the same motor-generator and pulse forming equipment. In view of the limited 
scale of the demonstration, no effort was made to use the full capability of the equipment.

The minimum equipment necessary for a TDEM receiver is a free-form induction loop consisting of a $160 \mathrm{~m}$ length of 25-26 conductor cable. In operation this cable was laid on the ground in to form a square. The conductors in the cable were connected in series so that, in effect, the cable had an area of $40000 \mathrm{~m}^{2}$ for detecting the time-rate of change of the vertical component of the magnetic field.

The voltage from this induction coil was connected directly to the input terminals of one channel of a Data Translation, Inc. Model 2805 analog to digital conversion board. The pertinent specifications for this piece of equipment are that it converts up to 8 channels of analog information to digital form at a maximum rate of 2500 samples per second. The digital information has a dynamic range of 12 bits, with the least bit having a value of approximately $10 \mu \mathrm{V}$.

The digital output of the A/D converter was stored directly in the hard-disk memory of a COMPAQ model SLT 286 portable computer. No filtering or amplification of the signal from the receiver coil was used. Subsequent data processing was carried out using the COMPAQ computer.

The generator was set up, tested and used to provide signals for observations at the first recording site, in a location known as Vignacce. Data logging was carried out using various sampling rates to find the best recording parameters. Similar recordings were made at three additional sites: Centro Capre, Poderone and Terzi di Sopra (fig. 1).

Recordings were made at each site using sampling rates of 50, 1000 and 2500 samples per second. All recordings were made with wide-band response; that is, no analog filtering was employed other than a damping capacitor across the induction loop. A segment of a recording made at a sampling rate of 2500 per second is shown in fig. 3.

Many time series were recorded of noise and signal plus noise at 4 locations within the «I Terzi» area. Each location was near a structure to which $50 \mathrm{~Hz}$ power was being delivered. The three time series shown in fig. 3 are all taken from a single noise recording made at the location known as Vignacce. The three traces shown in fig. 3 are plotted to three different time scales so that the short-term and long-term features of the ambient noise field can be seen. The noise is of significant amplitude. individual peaks reach amplitudes of 100 $\mathrm{mV}$, while the $50 \mathrm{~Hz}$ level appears to be \pm 25 $\mathrm{mV}$. Several power density spectrums computed from the recorded time series by Hartley transform are shown in fig. 4.

The spectrum labelled POWDENN1 was obtained from the time series for which portions were shown in fig. 3. This time series was sampled at 2500 samples per second, for which the Nyquist frequency is $1250 \mathrm{~Hz}$, as indicated by the frequency scale. The spectrum shows a large number of lines, of which the two most prominent appear at frequencies of $50 \mathrm{~Hz}$ and $800 \mathrm{~Hz}$. Other, lesser, spectral lines appear at nearly equal amplitudes at all the odd harmonics of $50 \mathrm{~Hz}, 150 \mathrm{~Hz}, 250 \mathrm{~Hz}, 350 \mathrm{~Hz}$, and so on, with the highest being $1250 \mathrm{~Hz}$.

The source of the line at $800 \mathrm{~Hz}$ is not known, though it is the strongest noise component seen at this site. It is probably an alias of a low frequency navigational transmitter. A small capacitor was used to shunt the receiving loop in order to suppress the higher frequencies in the spectrum POWDENN1 particularly those above the Nyquist frequency. The rollover frequency of the receiver loop was thus reduced to approximately $300 \mathrm{~Hz}$. The resulting spectrum for the noise at the same site is shown labelled as POWDENA1. The spectrum now appears to consist almost entirely of a $50 \mathrm{~Hz}$ component with its associated odd harmonics.

The spectrum labelled as POWDEN02 was recorded at a site known as Centro Capre. This site is a large building which contains the refrigerated storage equipment of the residences inside the «I Terzi» area. Operation of this equipment leads to a much stronger $50 \mathrm{~Hz}$ field, along with its odd harmonics, as well as strong spectral lines at frequencies of 200 and $400 \mathrm{~Hz}$. The spectrum labelled as POWDEN12 was recorded at a site known as Poderone. This site is a Club House wired for $50 \mathrm{~Hz}$ power 

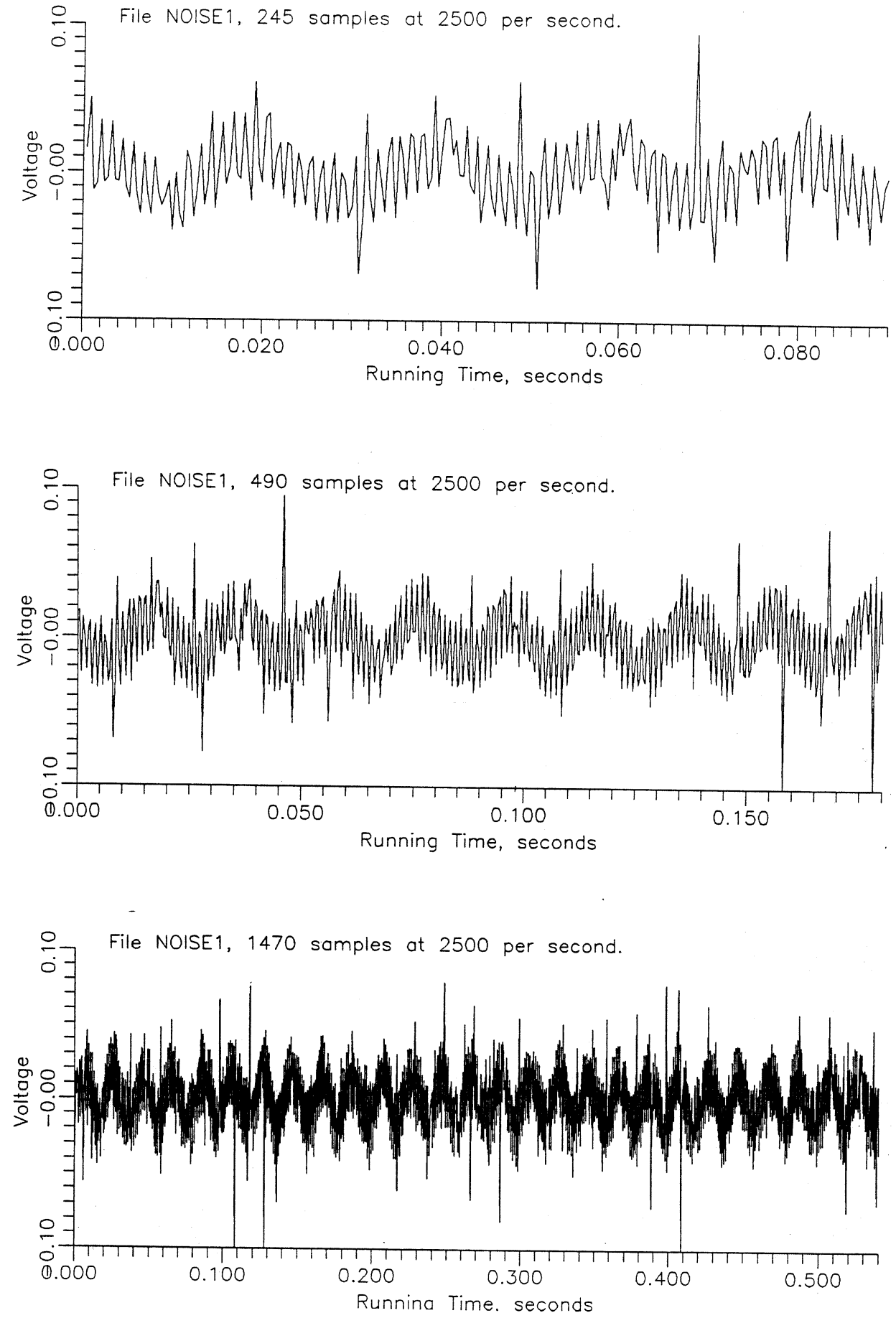

Fig. 3. Segment of one record. 

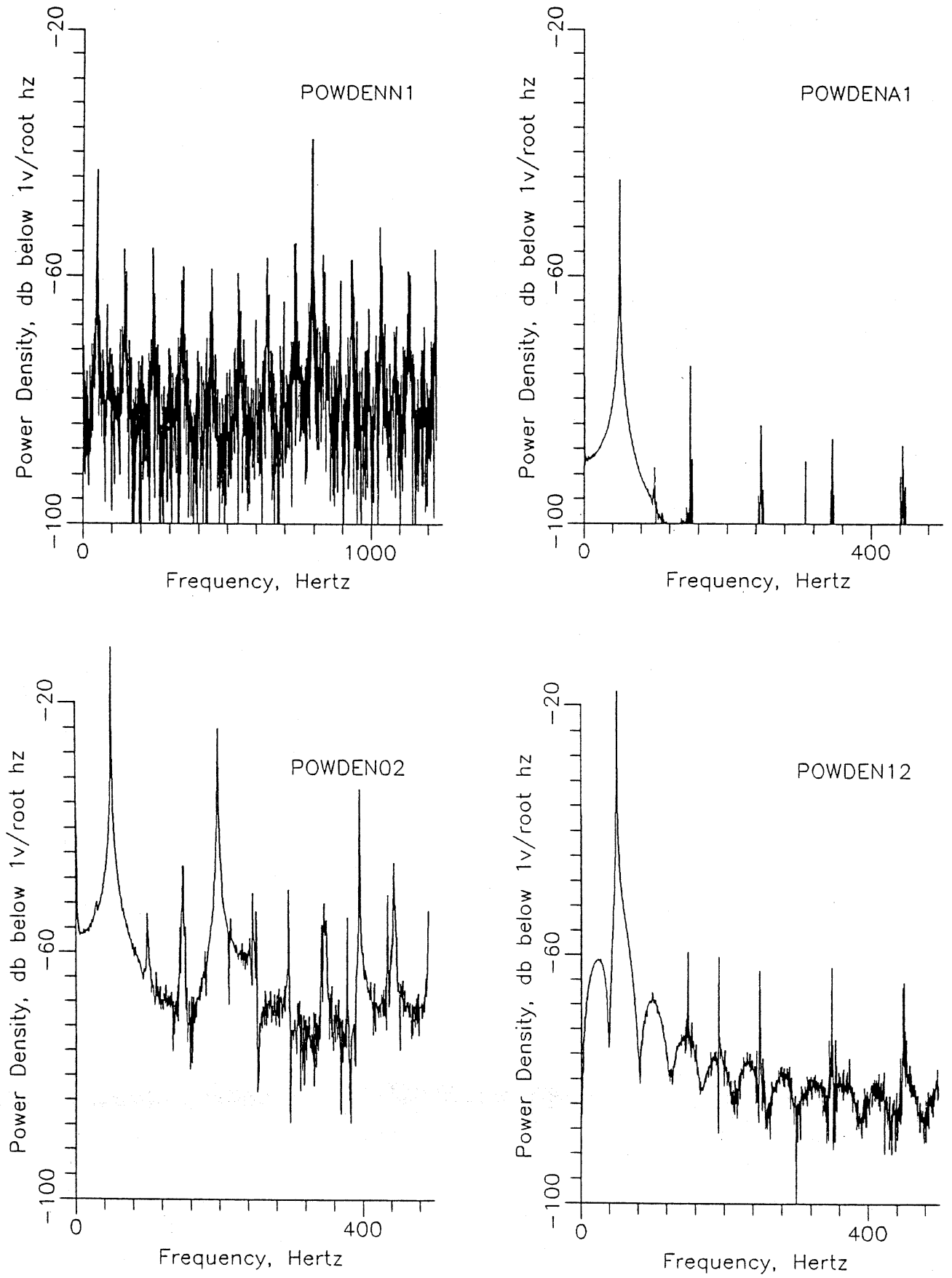

Fig. 4. Several power density spectrums. 
but with no power being used at the time of recording. The spectrum consists largely of $50 \mathrm{~Hz}$ with odd harmonics, and is quite similar to the spectrum POWDENA1, except that the amplitude is higher.

\section{Interpretation}

The signals were processed to suppress noise and reduce distortion introduced by the recording equipment. Specifically the source effect can be largely compensated by the simple process of self referencing of the observed signal.

In self referencing, the observed field (timederivative of the vertical component of the magnetic field) is referenced to the integral of the observed field. This integral is, of course, the DC magnetic field caused by the source. It is possible to compute the effective moment of the source simply from this integral by applying Gauss' law. This effective moment was then used in computing apparent resistivity.

The amount of source effect can easily be determined by comparing the effective source moment with the design source moment, which is the product of current and source length. For the measurements done from the «I Terzi» source, it was found that the effective source moment was 2.2 to $2.4 \%$ less than the design moment. This suggests that the apparent resistivity values calculated using self referencing were no more in error than 0.1 to $0.2 \%$ because of under-compensated source effects.

Now they are ready for conversion to an appropriate resistivity vs. depth profile. Inasmuch as we have sounding data from only a single location, interpretation is limited to a one dimensional model at best. Three dimensional interpretation requires the acquisition of a larger number of soundings.

Determination of a reasonable layered earth model to represent a set of TDEM sounding data is usually based on matching a TDEM curve computed for some model of the resistivity vs. depth function with the processed observed TDEM curve. Because TDEM curves are scalable, this comparison is best done in the Napierian domain.
The processed TDEM curve from fig. 5 was sampled in the Napierian domain at 20 equally spaced samples per decade, as shown in fig. 6 . The samples cover three decades in delay time, starting at $1 \mathrm{~ms}$. The points lying within the first $3 \mathrm{~ms}$ are flat, representing the fact that the deconvolution operator was determined over three points. The computation of TDEM curves from a piecewise constant model is straightforward. In a new area, the first step is usually to generate a starting model for an automatic optimization procedure. Inasmuch as we are dealing with a single TDEM curve, only the trial and error approach was used. In all, nine layered models were used to compute curves for comparison with the field data. The TDEM curves computed for the first three models are shown in fig. 7. The last two models, 8 and 9, superimposed on the sampled field curve, are shown respectively in figs. 8 and 9.

The final model deviates from the field data on the average by $2.35 \%$, point by point. Submission of the model and the data to an automatic inversion scheme results in only a minor

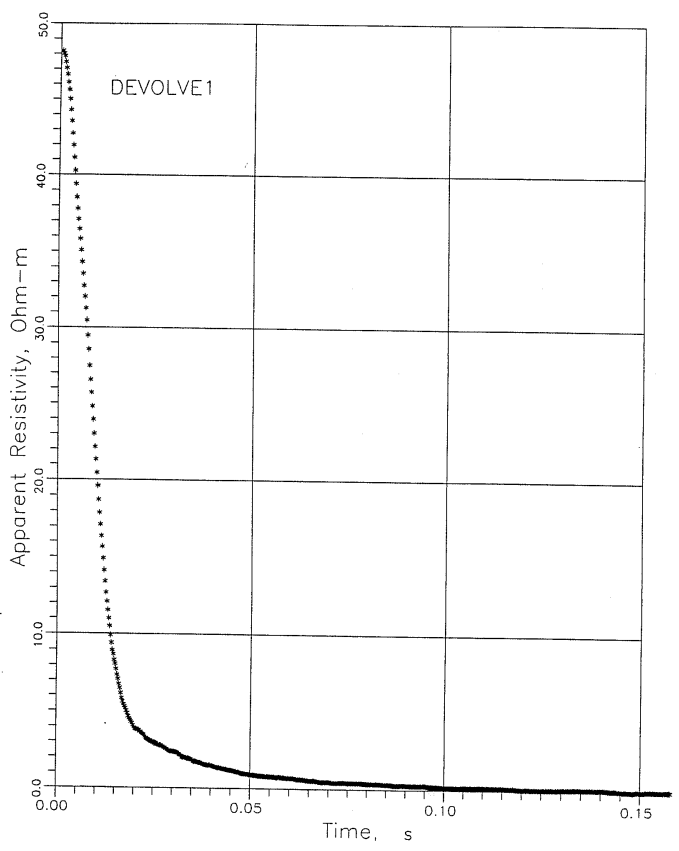

Fig. 5. Processed observed TDEM curve. 


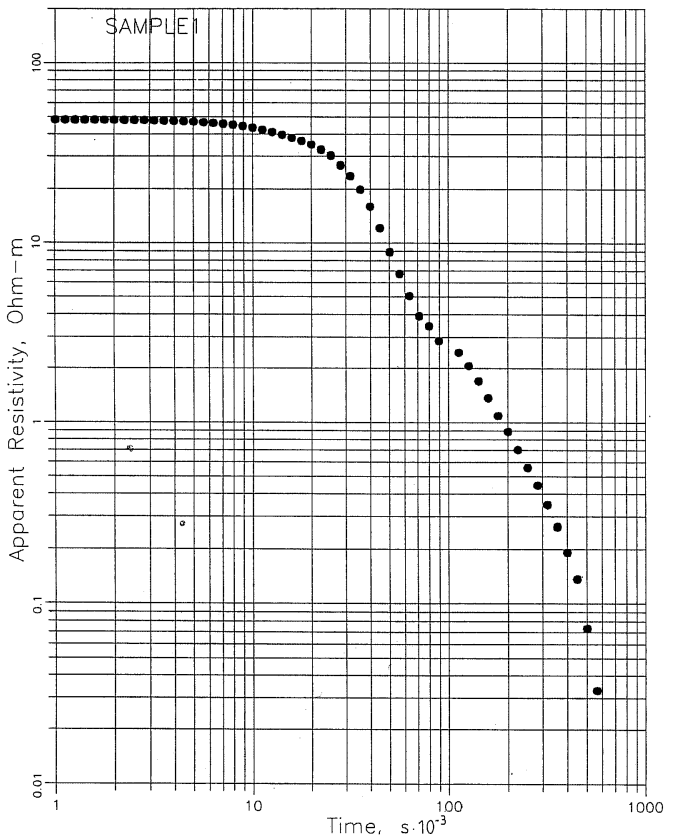

Fig. 6. Measured resistivity values.

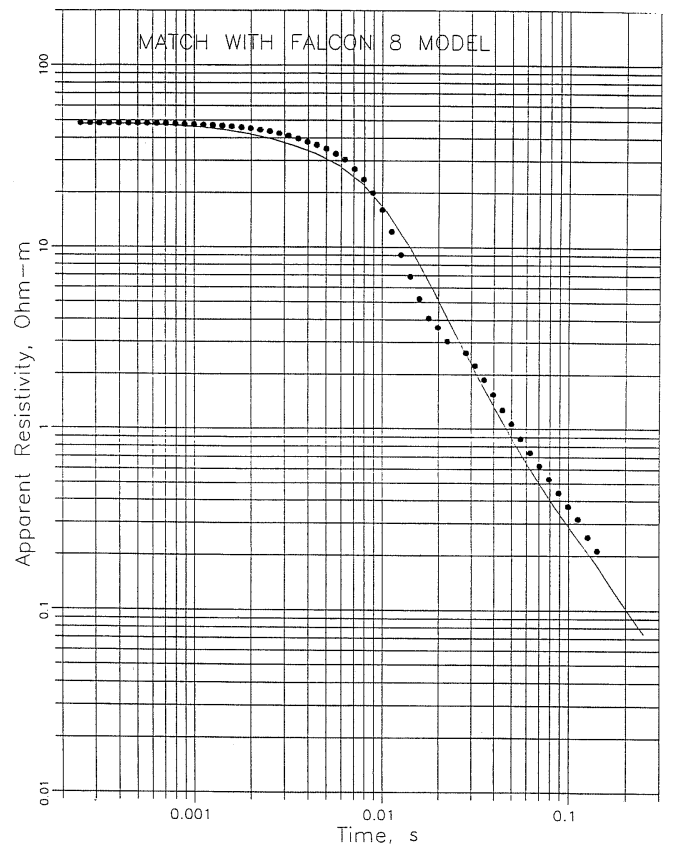

Fig. 8. 8 model calculated curve superimposed on the sampled field.

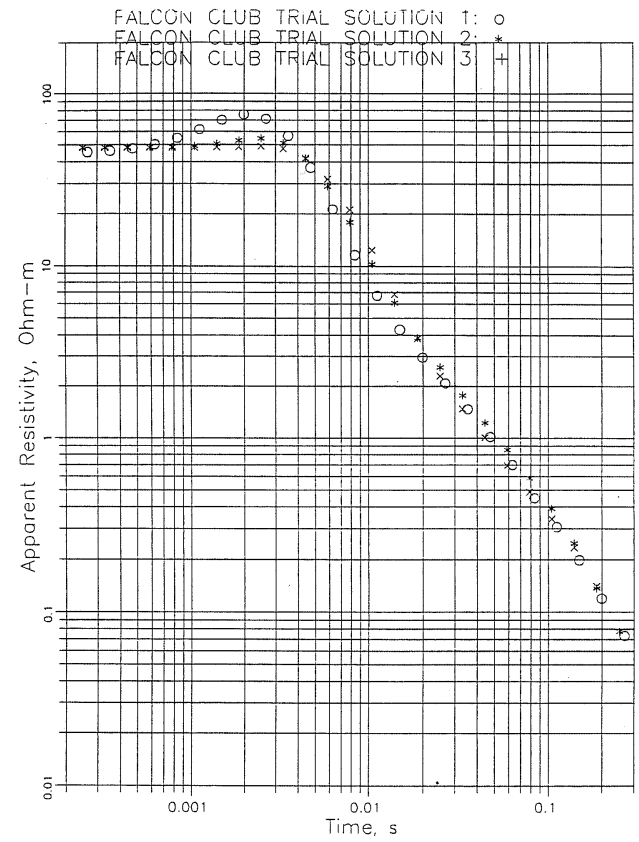

Fig. 7. Computed points for the first three models.

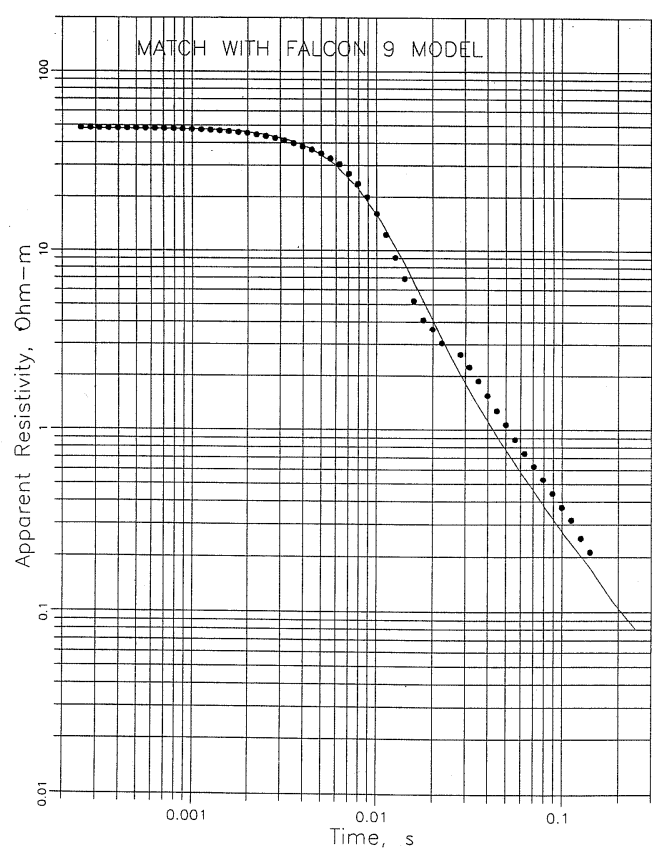

Fig. 9. 9 model calculated curve superimposed on the sampled field. 


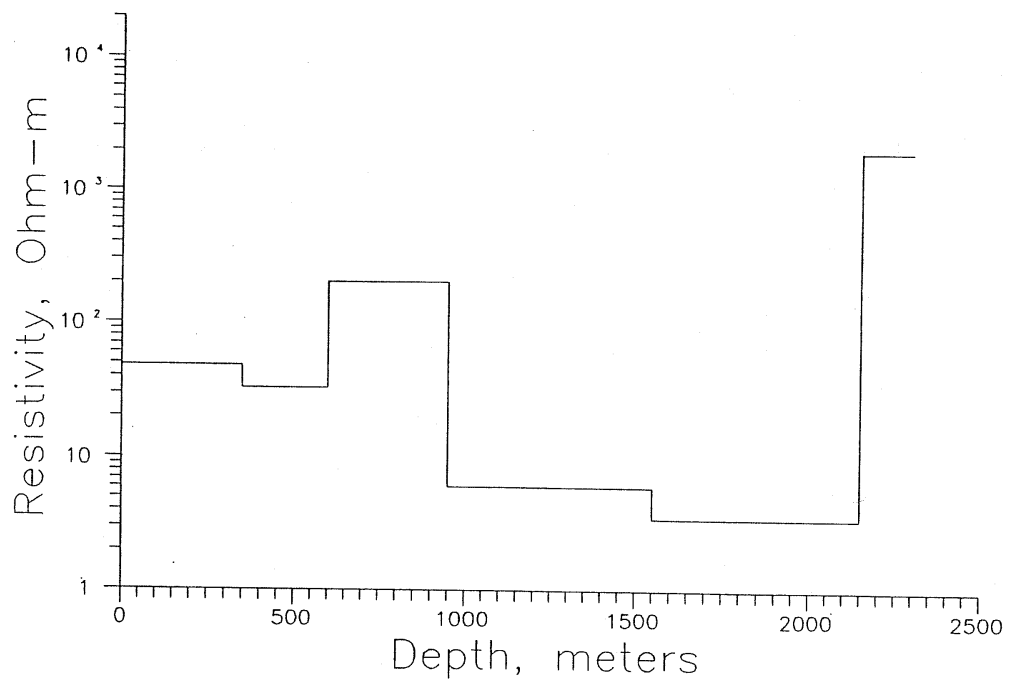

Fig. 10. Six layers model results.

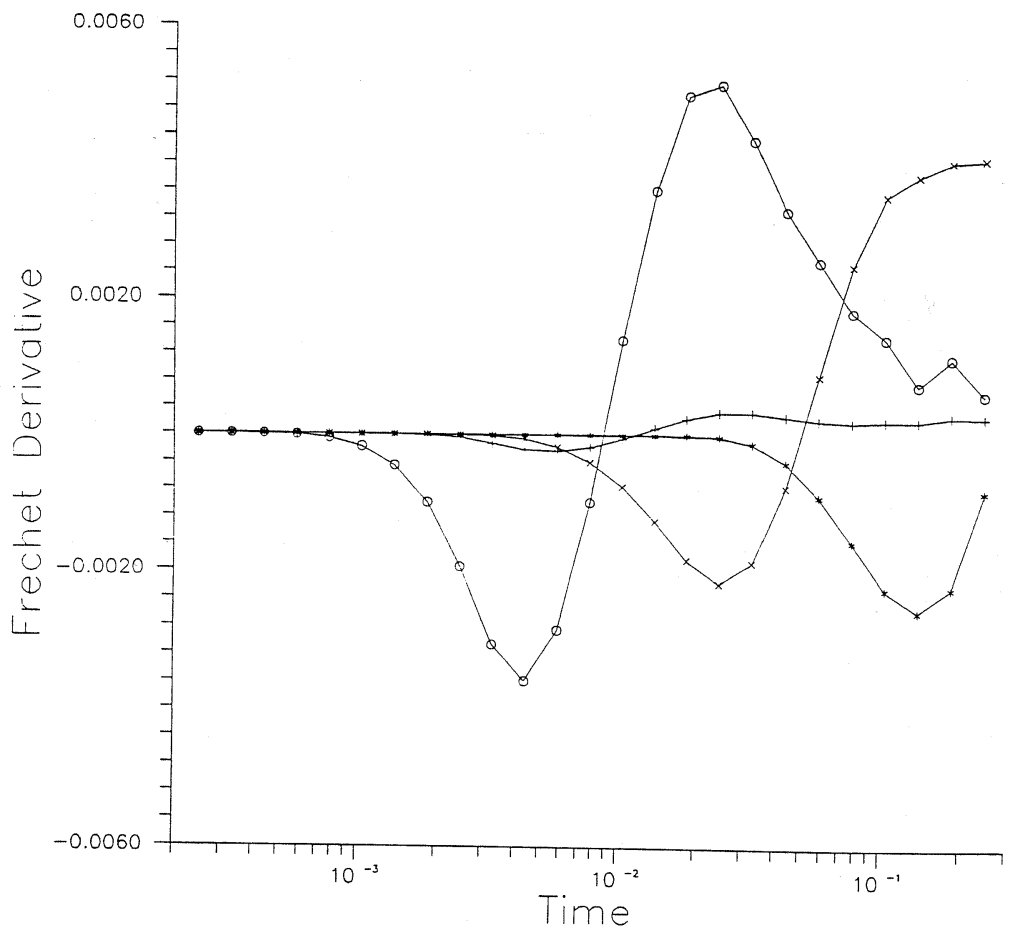

Fig. 11. Frechet derivative curve. 
reduction of the error of fit. This model would be a reasonable starting point for inversion of TDEM curves obtained at nearby sites, so that the trial and error estimation of a model would not be necessary.

The final model consists of six layers (fig. 10). We must examine the question as to whether this many layers can be resolved, or if the model has been over specified. This can be done by computed Frechet derivatives, derivatives of the theoretical TDEM curves with respect to the resistivity of one of the layers in the model. The Frechet derivative is a curve showing the change between the two models as a function of delay time. Frechet derivative curves were computed for the middle four layers of the six layer model. The results are shown in fig. 11. Layers 2, 4 and 5 show the Frechet derivative curves to be comparable in amplitude and shifted in time with respect to each other. Therefore, these three layers are resolvable from each other and moreover, the TDEM curve is equally responsive to each of three layers. Layer 3, a resistant layer, exhibits a Frechet derivative curve which is lower in amplitude that the other three, and which overlaps the response for the layer above and below, is much less resolvable. However, examination of models 1,2 and 3 (fig. 7) indicates that the presence or non presence of this layer has an obvious impact on the model curves.

\section{Conclusions}

The results obtained with a single TDEM sounding of less than normal precision cannot be used to draw profound conclusions about geological structures at «I Terzi», but they are quite significant in the projection of the results that would be obtained with a more extensive field effort.
The primary conclusion to be drawn is that environmental and geological conditions do not inhibit the use of the TDEM sounding method in areas like the «I Terzi». The required low contact resistance for the source cable can be obtained with little effort in soil such as that found along the Ombrone river in the investigated area. The effort required to obtain a grounding circuit capable of handing 200 A peak to peak current pulses is less than that met at most places where the TDEM sounding method has been used.

\section{Acknowledgements}

The authors are grateful to the ENEL S.p.A. for the most valuable technical support and to Mr. U. Viapiano, director of «Azienda Agricola Alta Maremma», for his pleasant supply support.

\section{REFERENCES}

HARTHILL, N.H. (1976): Time-Domain Electromagnetic Sounding, Inst. Elec. and Electron. Engineers Trans. Geosci. Elec., vol. GE-14, 256-260.

HördT, A., K.M. StRack, K. Vozoff and P.A. WolfGram (1990): Case histories of joint inversion of LOTEM and MT data, in 52nd EAEG Meeting in Kobenhavn (abstract vol.).

Kaufman, A. and G. V. Keller (1983): Frequency and Transient Soundings (Elsevier Science Publishing Co., Inc., Amsterdam), pp. 685.

Keller, G. V., J. I. Pritchardt, J.J. Jacobson and N.H. HARTHILL (1984): Megasource time-domain electromagnetic sounding methods, Geophysics, 49 (7), 9931009.

PetRY, H., K.M. STRACK and K. VozofF (1989): Inversion of LOTEM data, in Inverse Modeling in Exploration Geophysics - Proceedings of 6th Int. Math. Geophys., Seminar in Berlin 3-6 February 1988, edited by A. VoGEL, R. GORENFLÖ, B. KUMMER and C.O. OFOEGBU (Vieweg \& Sohn), 145-159. 